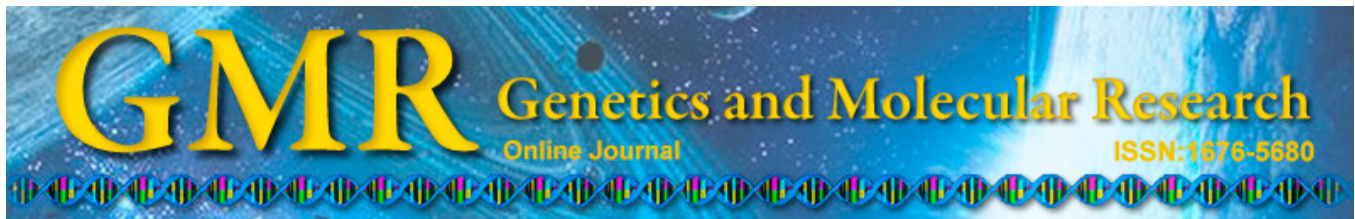

\title{
Co-culture of annulus fibrosus cells and bone marrow mesenchymal stem cells
}

\author{
Y. Xu' ${ }^{1 *}$, X.J. Zhang ${ }^{1 *}$, L. Fang ${ }^{2}$ and T.B. Zhao ${ }^{1}$ \\ ${ }^{1}$ Department of Orthopedics, West Hospital of Jinan Military General Hospital, \\ Shandong, China \\ ${ }^{2}$ Department of Orthopedics, Central Hospital of Shengli Oilfield, Dongying, \\ Shandong, China \\ *These authors contributed equally to this study. \\ Corresponding author: T.B. Zhao \\ E-mail: TingbaoZhao@163.com
}

Genet. Mol. Res. 14 (2): 3932-3938 (2015)

Received May 22, 2014

Accepted August 22, 2014

Published April 27, 2015

DOI http://dx.doi.org/10.4238/2015.April.27.7

\begin{abstract}
To investigate the effect on cell proliferation and extracellular matrix expression of annulus fibrosus (AF) cells when cocultured with bone marrow mesenchymal stem cells (BMSCs). Primary isolated rabbit $\mathrm{BMSCs}$ and $\mathrm{AF}$ cells were cultured and harvested cells were placed into a $15-\mathrm{mL}$ centrifugal tube co-culture system in a ratio of 2:1 (A group), 1:1 (B group), and 1:2 (C group). Cell proliferation was evaluated using cell counting kit-8, and mRNA of collagen II and mucopolysaccharide was quantified using real-time polymerase chain reaction (PCR) on Days 7, 14, and 21. The cell count, synthesized collagen II and mucopolysaccharide increased in a time-dependent manner, with a peak at Day 21. Cells in Group B proliferated faster and synthesized more collagen II and mucopolysaccharide than groups A and $\mathrm{C}$, where the difference was significant. AF cells and BMSCs in the ratio of $1: 1$, cultured using the centrifugal tube three-dimensional coculture system showed that BMSCs can promote AF cell proliferation
\end{abstract}


and extracellular matrix synthesis.

Key words: Intervertebral disc degeneration; Tissue engineering repair; Annulus fibrosus cell; Bone marrow mesenchymal stem cells; Three-dimension co-culture

\section{INTRODUCTION}

More than $20 \%$ of all low back pain (LBP) originates from degenerative changes in the intervertebral discs (IVDs), leading to progressive cell loss and degradation of the extracellular matrix (ECM) within the IVD (Wang et al., 2011). Disc degeneration due to age or injury is considered a major cause of LBP. Degeneration involves a series of biochemical and morphological changes, including decreased cell viability and proteoglycan synthesis leading to decreased water binding capacity. These changes result in loss of stability and flexibility, leading to back pain. Additionally, IVD degeneration is an irreversible phenomenon, which is often associated with LBP, imposing a great economic burden on health care and a substantial burden on society (Dagenais et al., 2008).

Despite this irreversible phenomenon, several techniques to decelerate disc degeneration have been reported. However, no technique has been proven effective to date for clinical application. Based on previous studies, we hypothesize that maintenance of proteoglycan content in the disc is achieved by avoiding depletion of the nucleus pulposus and that preserving the annulus structure is key to deceleration of disc degeneration (Sakai et al., 2003). One novel approach to prevent IVD degeneration is found at the stem cell level (Wang et al., 2010). Current studies have focussed on experimental animal models, to elucidate a specific biomechanics function within a physiological environment, however, the exact mechanism by which the IVD degenerates remains largely unknown (Smith et al., 2011).

Mesenchymal stem cells (MSCs) are known to possess the ability to differentiate into various kinds of cells from mesenchymal origin (Wei et al., 2009). Annulus fibrosus (AF) is an important component of the intervertebral disc and its critical role in prevention and treatment of IVD degeneration is associated with the integrity of structure and function of IVDs (Henriksson et al., 2009). Therefore, further study should be focused on the risk of IVD degeneration caused by stem cell transplantation.

The objectives of this study were to investigate the effect of co-culture of AF cells and BMSCs on cell proliferation and extracellular matrix expression of AF cells, so as to provide a foundation for a potential therapeutic model for disc degeneration.

\section{MATERIALS AND METHODS}

\section{Isolation and characterization of BMSCs}

Rabbit bone marrow-derived mesenchymal stem cells were isolated and characterized using the method described previously (Shi and Wang, 2010). In brief, Japanese white rabbit ( 3 weeks old) were anesthetized by auricular vein intravenous injection of $1 \%$ pentobarbital sodium and BMSCs were harvested by gently flushing the contents from tibias and femora with low-glucose Dulbecco's Modified Eagle's medium (1-DMEM; Gibco BRL, Gaithersburg, 
MD, USA). Density gradient centrifugation and adherent screening methods were used to isolate cultured BMSCs, which were then cultured in l-DMEM supplemented with $10 \%$ fetal bovine serum (FBS) and $20 \mathrm{U} / \mathrm{mL}$ penicillin-streptomycin in $5 \% \mathrm{CO}_{2}$ at $37^{\circ} \mathrm{C}$. The 3 rd-passage cells were prepared for flow cytometry (FCM) followed by incubation with surface antigens including goat anti-rabbit monoclonal antibodies (anti-CD29, anti-CD44, anti-CD105, anti-CD34, anti-CD45, anti-HLA-DR), which were labeled with fluorescein isothiocyanate (FITC)-conjugated rat anti-goat IgG. The isotypic antibody was used as negative control. All of the above antibodies were purchased from Santa Cruz Biotechnology, Inc. (Santa Cruz, CA, USA). Each surface antigen assay was performed in triplicate.

\section{Isolation and characterization of $\mathrm{AF}$}

The same animals used for BMSC isolation were anesthetized according to the method mentioned above. AF cells were isolated and characterized using the method described previously (Pan et al., 2012). Briefly, the rabbits were sacrificed, and the spine was removed and placed in culture medium. The AF cells were excised, marrow was removed, and AF cells were isolated, sectioned into small pieces $\left(1 \mathrm{~mm}^{3}\right)$, and digested enzymatically for $45 \mathrm{~min}$ with $0.25 \%$ trypsin and $0.03 \%$ collagenase in Hank's balanced salts solution. After digestion, cells were pelleted at $800 \mathrm{RPM} / \mathrm{min}$ in a Beckman TJ-6 centrifuge for $5 \mathrm{~min}$, the supernatant was removed, and cells were resuspended in $5 \mathrm{~mL}$ DMEM/F12 containing 10\% FBS and $100 \mathrm{units} / \mathrm{mL}$ penicillin/streptomycin. Cells were dissociated by repeatedly passing them through a narrowed Pasteur pipette, then added to a laminin-coated $35-\mathrm{mm}$ dish, and incubated at $37^{\circ} \mathrm{C}$ under $5 \% \mathrm{CO}_{2}$ for 3 days. The 3rd-passage cells, assessed for type II collagen and alkaline phosphatase expression by immunohistochemistry, were used for characterization of AF. Meanwhile, the optimal passages of AF were selected for co-cultures using MTT assay.

\section{Co-culture of BMSCs and AF}

According to the method described previously (Le Visage et al., 2006) for co-culture pellets, $5 \times 10^{5}$ cells in $1 \mathrm{~mL}$ culture medium (DMEM + 10\% FBS) were centrifuged at $240 \mathrm{~g}$ for $5 \mathrm{~min}$ to form pellets and were seeded onto $15 \mathrm{~mL}$ centrifuge tubes (co-culture group) in varying proportions (1:2 for Group A; 1:1 for Group B; 2:1 for Group C), followed by 5-h incubation at $37^{\circ} \mathrm{C}$ under $5 \% \mathrm{CO}_{2}$. Meanwhile, the control group including BMSCs and $\mathrm{AF}$ cultures were also cultured in regular medium for 1, 2, and 3 weeks and all groups were used for further analysis.

\section{Cell proliferation assay}

At various time-points, tissues were digested overnight using papain and cell suspensions were prepared according to the method mentioned above. Cell suspensions were also added to 96-well plates and cell proliferation was determined by analyzing expression using CCK-8 method.

\section{Real-time PCR}

At various time-points, total RNA was extracted using Trizol reagent (Invitrogen, Carlsbad, CA, USA) and the expression of Aggrecan mRNA was analyzed by real-time poly- 
merase chain reaction (RT-PCR). The primers (Table 1) were designed using the Primer5 5.0 software and synthesized by Invitrogen Co.ltd. RT-PCR for quantitative analysis of changes in aggrecan expression was replicated 4 times and band density (gray scale) was normalized to that of $\beta$-actin. The reactions were performed using the SYBR PrimeScript RT-PCR kit (Takara Bio, Inc.) with an ABI 7500 Real-Time PCR System (Applied Biosystems) according to the standard protocol. As an internal control, levels of $\beta$-actin were quantified in parallel with the target genes. Crossing threshold $\left(\mathrm{C}_{\mathrm{t}}\right)$ values were calculated by the 7500 Fast Real-Time PCR System software using the second derivative maximum method. Data were analyzed using the $2^{-\triangle \Delta \mathrm{Ct}}$ method. All samples were run in triplicate.

\section{Statistical analysis}

Statistical analyses were conducted using the Statistical Package for Social Sciences System Version 14.0 (SPSS, Chicago, IL, USA). Nonparametric data (Pfirrmann grading and histological grading) were analyzed by the Kruskal-Wallis $H$ test. Gene expression changes at different observed times were analyzed by One-way ANOVA test. A P value of $<0.05$ was considered to be statistically significant.

\section{RESULTS}

\section{Basic characteristics of BMSCs}

Primarily cultured BMSCs attained confluence after 5 to 7 days. The 3rd-passage cells showed a high growth rate and conspicuous syntropic characteristics. The results of cell surface antigen detection showed that the identification markers of mesenchymal cell such as CD29, CD44, and CD105 decreased on BMSCs. Meanwhile, BMSCs expressed CD34, CD45, and HLA-DR, which are the identification markers of hematopoietic cell surface antigen (Figure 1).

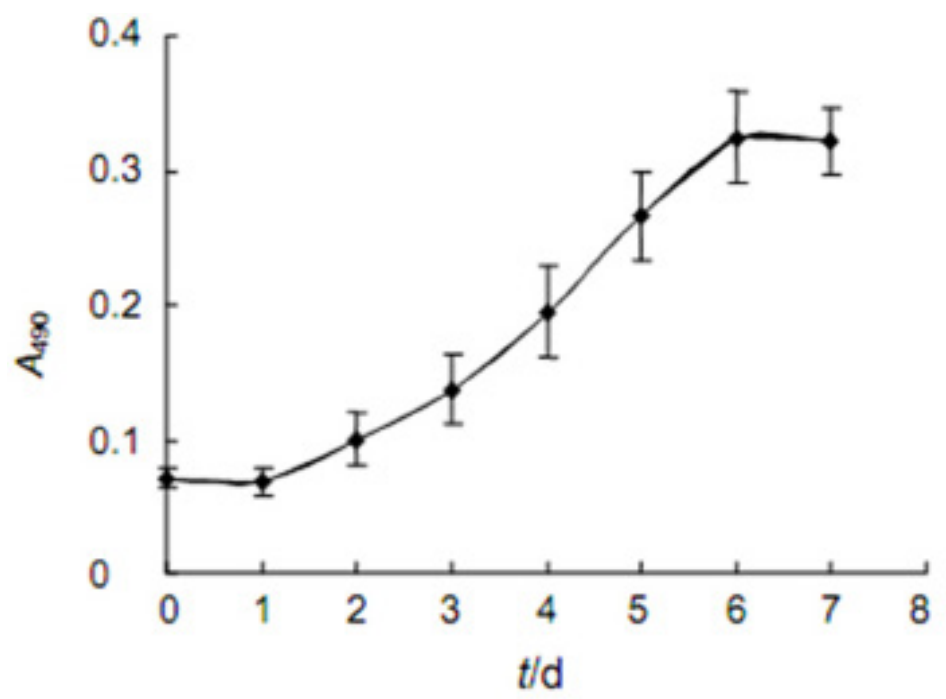

Figure 1. Growth curve of isolated and cultured annulus fibrosus cells. 


\section{Basic characteristics of AF}

Morphology characterization showed that the adherent cells were shaped as polygons and short fusiforms. Using immunohistochemistry staining, cells positive for expressed type II collagen appeared with brown cytoplasm, but without staining of the nucleus. As shown in Figure 1, cytoplasm of positive cells were covered with sepia particles and the growth curve of isolated and cultured annulus fibrosus cells showed a 4-day exponential growth phase, at which stage they were used for subsequent analyses during the 3rd passage.

\section{Basic characteristics of co-cultured BMSCs and AF}

After 3 days of BMSCs and AF co-culture, inner cell mass (ICM) rose gradually, however the maximum volume was attained at Day 21, observed in Group B in contrast to groups A and C. Using hematoxylin-eosin (HE) staining, the arrangement of cells in Group B showed greater compactness than groups $\mathrm{A}$ and $\mathrm{C}$.

\section{Proliferation of co-culture of BMSCs and AF}

Cell proliferation after co-culture of BMSCs and AF was analyzed using CCK-8 assay. As shown in Table 1, there was no cell proliferation in all groups at Day 7 of co-culture. At Day 14, cell proliferation was significantly higher in Group B than that in groups A and C, with no significant difference between cell proliferation in groups A and C. Additionally, though cell proliferation was significantly increased with successive co-culture for 3 weeks, there was no significant difference between cell proliferation in groups A and C.

Table 1. Proliferative comparison of BMSCs and AF cells in different proportion combined groups (means $\pm \mathrm{SD}$ ).

\begin{tabular}{|c|c|c|c|c|}
\hline \multirow[t]{2}{*}{ Groups } & \multirow[t]{2}{*}{$\mathrm{N}$} & \multicolumn{3}{|c|}{ OD 490} \\
\hline & & 7 days & 14 days & 21 days \\
\hline $\mathrm{A}$ & 6 & $0.25 \pm 0.04$ & $0.35 \pm 0.07 *$ & $0.43 \pm 0.13^{\#}$ \\
\hline B & 6 & $0.28 \pm 0.06$ & $0.45 \pm 0.06^{* \boldsymbol{\Delta}}$ & $0.58 \pm 0.15^{\#}$ \\
\hline $\mathrm{C}$ & 6 & $0.27 \pm 0.05$ & $0.38 \pm 0.08^{*}$ & $0.45 \pm 0.11^{\#}$ \\
\hline
\end{tabular}

*Compared to 7d, P < 0.05; \#compared to $14 \mathrm{~d}, \mathrm{P}<0.05 ;{ }^{\wedge}$ compared to groups $\mathrm{A}$ and $\mathrm{C}, \mathrm{P}<0.05$.

\section{Expression of Aggrecan mRNA and type II collagen}

As shown in Table 2, at day 21 of BMSCs and AF co-culture, expression of Aggrecan mRNA and type II collagen was significantly increased in Group B in contrast to groups A and $\mathrm{C}$, with no significant difference between the groups $\mathrm{A}$ and $\mathrm{C}$.

$\begin{aligned} & \text { Table 2. Comparison of collagenase II and mucopolysaccharide in different BMSCs and AF proportion } \\
& \text { combined group (means } \pm \text { SD). }\end{aligned}$
\begin{tabular}{lccc}
\hline Groups & $\mathrm{N}$ & mRNA \\
\cline { 2 - 3 } & & Type II collagen & Glycosaminoglycans \\
\hline A & 6 & $0.19 \pm 0.11$ & $0.40 \pm 0.21$ \\
B & 6 & $3.88 \pm 0.26^{\mathbf{\Delta}}$ & $4.45 \pm 0.39 \mathbf{\Delta}$ \\
C & 6 & $0.21 \pm 0.13$ & $0.43 \pm 0.24$ \\
\hline
\end{tabular}

${ }^{4}$ Compared to groups A and $\mathrm{C}, \mathrm{P}<0.01$. 


\section{DISCUSSION}

Degenerative disc disease (DDD) is a common disease, accompanied by biochemical changes in the degenerated disc, including dehydration, decrease of osmotic pressure, quantitative and distributive changes in collagen, decreased proteoglycan content, and downregulated proteoglycan expression (Yang et al., 2009). The current strategy of conservative and surgical treatment can alleviate clinical symptoms, but is limited to using novel biological solutions and dynamic stabilization (Sakai et al., 2003). Therefore, significant efforts are urgently required for the development of effective therapy for IVD degeneration.

Mesenchymal stem cells (MSCs) isolated from bone marrow aspirates provide an almost unlimited source of cells with extremely high proliferation activity and the potential to differentiate into several mesenchymal cell lineages, including chondrogenic differentiation (Allon et al., 2010), making it plausible to delay disc degeneration using intradiscal transplantation of MSCs. Observation of biological activity has shown that BMSC phenotypes express several CD markers including CD44, CD105, and CD166, following addition of BMSCs and sodium alginate gel in different culture conditions (Allon et al., 2010).

Aggrecan fragmentation and loss is a hallmark of disc degeneration, occurring early in the degenerative process as well as in normal ageing and is commonly used as a marker of disc degeneration. Loss of aggrecan can be due to either an increase in proteinase production or a decrease in aggrecan synthesis, resulting in the decreased number of IVDs and ability of biologic repair of disc degeneration in the advanced stage, which indicate that AFC could be considered as a target cell for repair of IVD degeneration (Miyamoto et al., 2010; Longo et al., 2012). Therefore, in order to address these issues, we propose that cell transplantation of a co-culture of BMSCs and AF could be used for IVD degeneration repair.

A 3-D model of BMSC and AF co-culture has been established in previous studies and the feasibility was also verified, showing high purity, proliferation and biological security, demonstrating that BMSC could promote the expression of type II collagen and proteoglycans in AF cells (Richardson et al., 2006). Accordingly, we investigate the effect of AF cell and BMSCs co-culture on AF cell proliferation and extracellular matrix expression using a centrifugal tube three-dimension co-culture system. This is ideally suited to the tissue from which the extracellular matrix (ECM) is harvested, which allows ECM scaffolds to promote constructive remodeling similar to naturally evolved mechanisms of tissue homeostasis, repair, and replacement following injury (Badylak et al., 2009). Our study has shown that the cell crumb profiteered and promoted collagen II and mucopolysaccharide synthesis in a time-dependent manner with a peak at day 21 which may be related to some factors including the interaction between cell space and cytokines (Abdallah and Kassem, 2009).

Due to the increasing evidence that transplanted BMSCs could secrete cytokines including TGF- $\beta 1$, IGF-1, EGF, and PDGF, AF could adapt to the growth environment including downregulated nutrition, leading to the arrest of the cell cycle (Abdallah and Kassem, 2009). Therefore, in this study, rabbit BMSCs and AF cells were primary isolated and cultured in varying ratios, cell proliferation was evaluated using CCK-8 method, and collagen II and mucopolysaccharide mRNA expression were quantified. Our results have shown that the optimal ratio for BMSCs and AF cell co-culture (1:1) is conducive to growth of both cell types, due to growth factor(s) induced by co-culture and an activated potential signaling involved in the synthesis of type II collagen and glycosaminoglycans. 
In conclusion, the present study mimics the natural interaction between BMSCs and $\mathrm{AF}$ in the AF cells and BMSCs centrifugal tube three-dimension co-culture system in the ratio of 1:1, which promotes AF cell proliferation and extracellular matrix synthesis, providing a novel therapeutic strategy for IVD degeneration.

\section{Conflicts of interest}

The authors declare no conflict of interest.

\section{REFERENCES}

Abdallah BM and Kassem M (2009). The use of mesenchymal (skeletal) stem cells for treatment of degenerative diseases: current status and future perspectives. J. Cell Physiol. 218: 9-12.

Allon AA, Aurouer N, Yoo BB, Liebenberg EC, et al. (2010). Structured coculture of stem cells and disc cells prevent disc degeneration in a rat model. Spine J. 10: 1089-1097.

Badylak SF, Freytes DO and Gilbert TW (2009). Extracellular matrix as a biological scaffold material: Structure and function. Acta Biomater. 5: 1-13.

Dagenais S, Caro J and Haldeman S (2008) A systematic review of low back pain cost of illness studies in the United States and internationally. Spine J. 8: 8-20.

Henriksson HB, Svanvik T, Jonsson M, Hagman M, et al. (2009). Transplantation of human mesenchymal stems cells into intervertebral discs in a xenogeneic porcine model. Spine (Phila Pa 1976) 34: 141-148.

Le Visage C, Kim SW, Tateno K, Sieber AN, et al. (2006) Interaction of human mesenchymal stem cells with disc cells: changes in extracellular matrix biosynthesis. Spine (Phila Pa 1976) 31: 2036-2042.

Longo UG, Papapietro N, Petrillo S, Franceschetti E, et al. (2012). Mesenchymal stem cell for prevention and management of intervertebral disc degeneration. Stem Cells Int. 2012: 921053.

Miyamoto T, Muneta T, Tabuchi T, Matsumoto K, et al. (2010). Intradiscal transplantation of synovial mesenchymal stem cells prevents intervertebral disc degeneration through suppression of matrix metalloproteinase-related genes in nucleus pulposus cells in rabbits. Arthritis Res. Ther. 12: R206.

Pan Y, Chu T, Dong S, Hao Y, et al. (2012). Cells scaffold complex for Intervertebral disc Anulus Fibrosus tissue engineering: in vitro culture and product analysis. Mol. Biol. Rep. 39: 8581-8594.

Richardson SM, Walker RV, Parker S, Rhodes NP, et al. (2006) Intervertebral disc cell-mediated mesenchymal stem cell differentiation. Stem Cells 24: 707-716.

Sakai D, Mochida J, Yamamoto Y, Nomura T, et al. (2003). Transplantation of mesenchymal stem cells embedded in Atelocollagen gel to the intervertebral disc: a potential therapeutic model for disc degeneration. Biomaterials 24: 3531-41.

Shi ZB and Wang KZ (2010). Effects of recombinant adeno-associated viral vectors on angiopoiesis and osteogenesis in cultured rabbit bone marrow stem cells via co-expressing hVEGF and hBMP genes: a preliminary study in vitro. Tissue Cell 42: 314-321.

Smith LJ, Nerurkar NL, Choi KS, Harfe BD, et al. (2011). Degeneration and regeneration of the intervertebral disc: lessons from development. Dis. Model Mech. 4: 31-41.

Wang F, Wu XT, Zhuang SY, Wang YT, et al. (2011). Ex vivo observation of human nucleus pulposus chondrocytes isolated from degenerated intervertebral discs. Asian Spine J. 5: 73-81.

Wang YT, Wu XT and Wang F (2010). Regeneration potential and mechanism of bone marrow mesenchymal stem cell transplantation for treating intervertebral disc degeneration. J. Orthop. Sci. 15: 707-719.

Wei A, Tao H, Chung SA, Brisby H, et al. (2009). The fate of transplanted xenogeneic bone marrow-derived stem cells in rat intervertebral discs. J. Orthop. Res. 27: 374-379.

Yang F, Leung VY, Luk KD, Chan D, et al. (2009). Mesenchymal stem cells arrest intervertebral disc degeneration through chondrocytic differentiation and stimulation of endogenous cells. Mol. Ther. 17: 1959-66. 